\title{
Preputial Stones as a Rare Complication of Buried Penis: A Case Report
}

\author{
Si Hyun Kim, Doo Sang Kim \\ Department of Urology, Soonchunhyang University Cheonan Hospital, Soonchunhyang University College of Medicine, Cheonan, Korea
}

\begin{abstract}
Preputial calculi are a very rare manifestation of urolithiasis that occur most often in uncircumcised elderly males with poor hygiene and low socioeconomic status. Here, we present a case of preputial calculi in a 13-year-old boy with a buried penis. No penile shaft was observed and hard materials palpated around the penile foreskin. Imaging examinations showed several stones around the penis and surgical correction was indicated. The stones were removed and a circumferential incision along the coronal edge allowed for degloving with rotated skin flaps. Surgical removal of the stones and reconstruction was performed to promote hygiene and prevent future infection.
\end{abstract}

Keywords: Urinary calculi; Penis; Phimosis; Reconstructive surgical procedures; Case report

\section{INTRODUCTION}

A buried penis occurs when the penis does not have a suitable skin coat and remains buried under the skin of the abdomen, thigh, or scrotum. This condition, also referred to as hidden penis, inconspicuous penis, and concealed penis, is associated with psychological problems, local infection, urinary retention, voiding difficulty, and sexual problems [1,2]. Our patient had large stones in the prepuce; however, the buried penis made long-term care and management difficult. To the best of our knowledge, this is the first case report of a buried penis with preputial stones, a very rare form of urolithiasis in uncircumcised patients [3].

This case report was conducted in accordance with the Declaration of Helsinki. Written informed consent was obtained from the patient for publication of this report and any accompanying images. The authors have prepared and revised this manuscript according to the CARE Checklist.

\section{CASE REPORT}

A 13-year-old boy visited Soonchunhyang University Cheonan Hospital with pain and redness around the genitals. The patient with hypoxic brain damage arrived at our hospital with his mother and complained of redness and pain around the genitals. According to his mother, he experienced penis pain during diaper changes and redness in the surrounding area. She had not previously been aware of these symptoms, and initially thought it was diaper rash. However, the patient's symptoms did not improve for about 2 months.

The patient had complex partial status epilepticus due to hypoxic brain damage caused by meconium aspiration syndrome. He had hypothyroidism and quadriplegia and had undergone percutaneous endoscopic gastrostomy tube insertion. His mother said that he had diaper voiding and that his urine contained impurities, like sand dust. There were no other complaints about urination. The patient had no specific family history.

The patient had normal vital signs. Redness was detected around the opening of the penis; the penis shaft was not observed (Fig. 1A). The affected area was warm and characterized by hard palpable masses that produced audible sounds similar to stones clashing. The testicles were palpated in the inguinal area. The white blood cell (WBC) count was slightly elevated, but other blood test results were normal. The dominant WBC type was neutrophils (57\%).

A plain film was taken prior to surgery. A radio-opaque shadow 

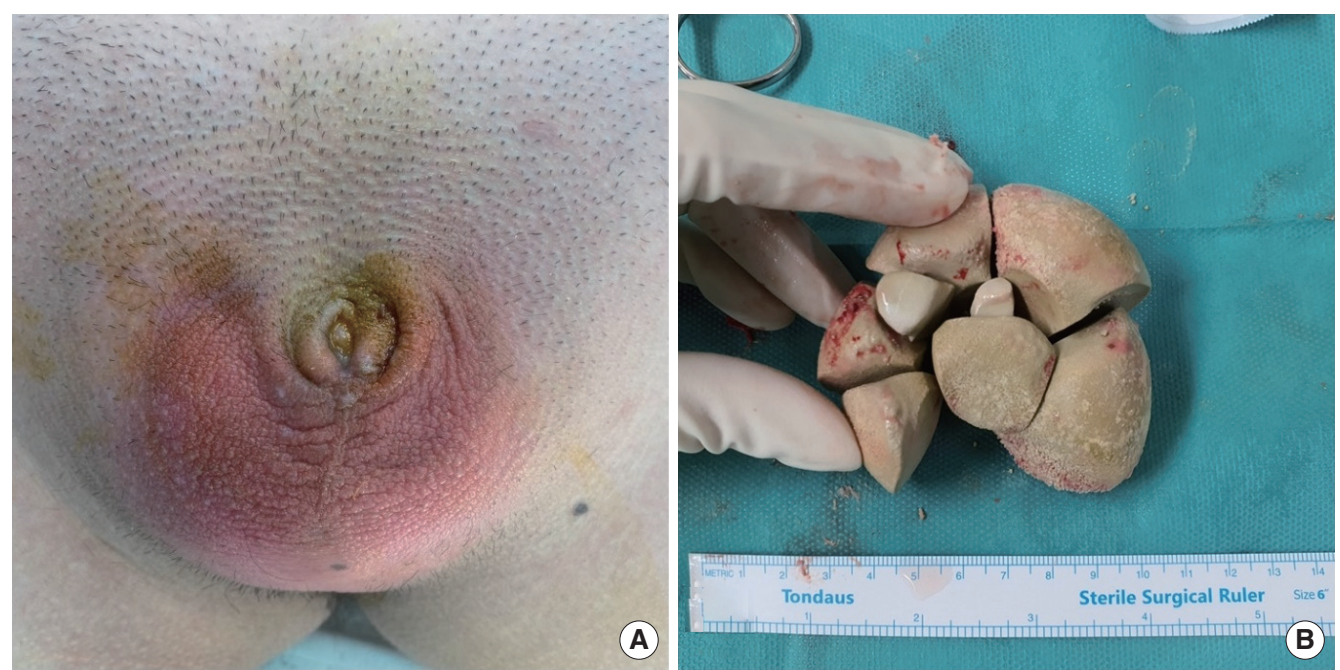

Fig. 1. (A) A preoperative photograph showing redness around the orifice. (B) A photograph of the extracted stones.
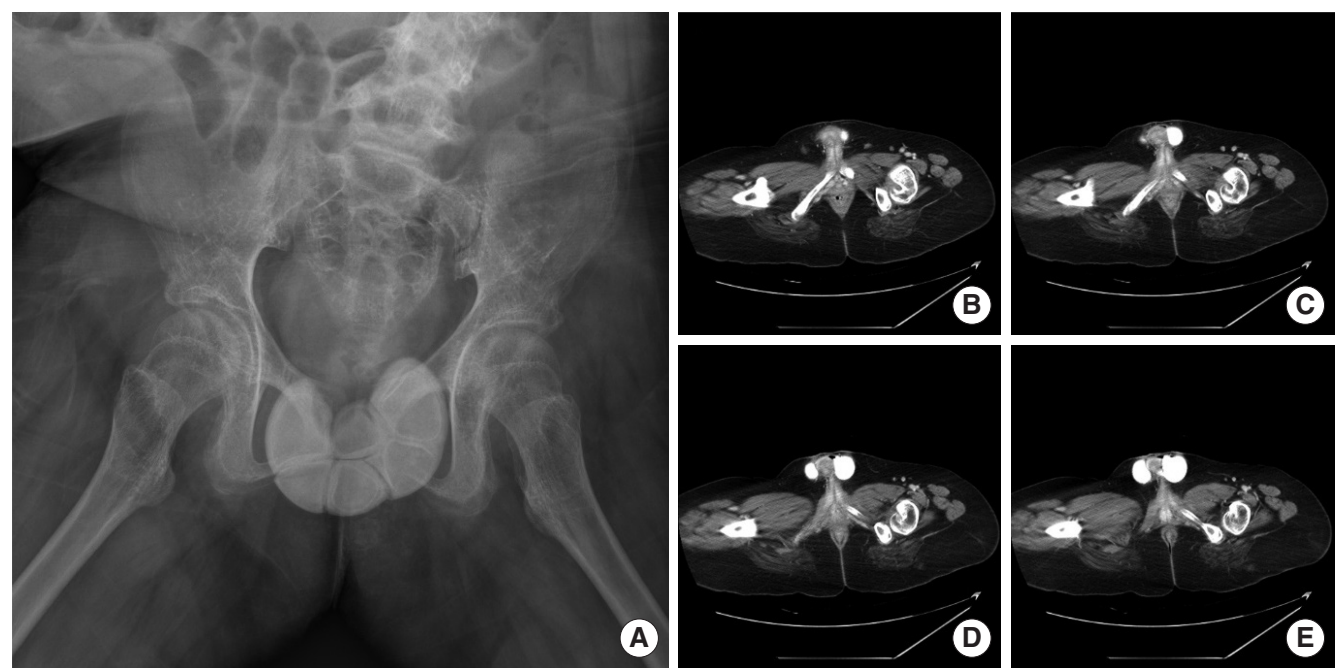

Fig. 2. (A) A plain film showing radio-opaque materials around the penis. (B-E) An enhanced computed tomography image showing the margin between surrounding tissues and the stones.

resembling an orange segment, presumed to be a stone, was observed around the buried penis (Fig. 2A). Enhanced computed tomography (CT) was performed to determine if there was an association with the corpus cavernosum or corpus spongiosum. Highdensity materials with clear boundaries surrounding the penile body were observed (Fig. 2B-E). The final diagnosis was a buried penis with preputial stones.

\section{Treatment}

The operation was performed under general anesthesia. The patient was placed in the supine position and prepared in the normal manner using povidone and disposable surgical drapes. The surgery started with a midline longitudinal skin incision on the ventral surface, from the penoscrotal junction to the tip of the foreskin. After the incision, multiple stones were observed between the glans penis and penile skin, in the preputial space. The stones were removed (Fig. 1B) and urethral catheterization was performed using a $16 \mathrm{~F}$ catheter to protect the urethra. A circumferential incision was made $1 \mathrm{~cm}$ below the glans penis. Degloving of the penile skin was performed up to the penoscrotal junction. The skin and subcutaneous fibrous tissue around the penile shaft were excised. The tunica albuginea at the base of the shaft was fixed to the prepubic fascia at the 2 and 10 o'clock positions using 4-0 Prolene sutures. After adjusting the median line on the ventral 

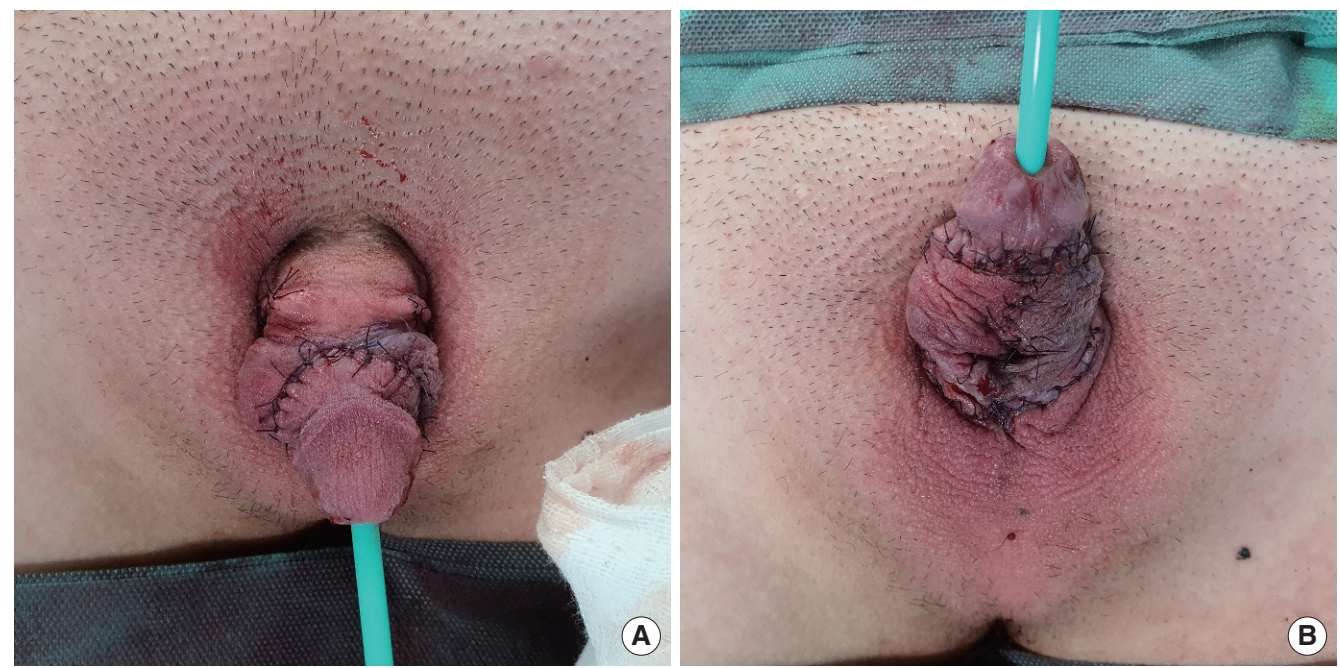

Fig. 3. (A, B) A postoperative photograph.

side, the excess skin was incised and sutured. The operation was terminated after suturing the circumcision line (Fig. 3).

\section{Outcome and follow-up}

All stitches were removed on postoperative day 8. Two months after the operation, the patient was admitted to the pediatrics department with pneumonia. Overall, the patient's mother is very satisfied with the outcome of the surgery and the patient no longer complains of discomfort.

\section{DISCUSSION}

A buried penis, also known as a hidden or concealed penis, occurs when the penile shaft is hidden inside the suprapubic fat pad [4]. It results from poor fixation at the base of the penis, obesity, or scarring after penile surgery. The patient described in this study had a congenital buried penis, where the penile shaft is not fixed to the deep fascia and is pushed inside.

Known complications of a buried penis include difficulty voiding, infection, and psychosocial problems [2,5]. However, preputial stones are uncommon and typically occur in adult males with phimosis [6]. The pathogenesis of preputial calculi remains incompletely understood, but they are known to arise from inspissated smegma, struvite formation secondary to an infection, and disrupted migration of stones formed in the proximal urinary tract [7]. Carbonate, ammonia, magnesium, and phosphate were detected in the stones extracted from our patient. Thus, we de- duced that the stones were calcium phosphate stones and magnesium ammonium phosphate stones [8]. Calcium phosphate stones are the second most common stones found in the urinary tract and are most likely caused by the accumulation of urine inside the prepuce. Magnesium ammonium phosphate stones are most likely caused by infection.

There are various surgical techniques for treating buried penis [9]. In this case, the method published by Manasherova et al. [10] was applied. A midline longitudinal incision was made up to the ventral surface, from the penoscrotal junction to the preputial skin. Then, degloving was performed using a rotation flap were performed. This method was used because of the longitudinal incision removal of large stones from the preputial space and incision and reconstruction of the excess skin after stone removal.

In summary, we have outlined the diagnosis and treatment of a rare complication of a buried penis. Our study was limited by the short follow-up period, and the inclusion of a patient with many comorbidities. However, this case report is important because it describes a situation not typically encountered in daily clinical practice.

In conclusion, preputial stones in patients with a buried penis are rare. However, as demonstrated by this case, they can develop in unique circumstances. In patients with buried penis and preputial stones, surgical correction is the best treatment option to improve hygiene and prevent infection. 


\section{CONFLICT OF INTEREST}

No potential conflict of interest relevant to this article was reported.

\section{ACKNOWLEDGMENTS}

This manuscript was checked by professional editors with English as their first language. This work was supported by the Soonchunhyang University Research Fund.

\section{ORCID}

\author{
Si Hyun Kim https://orcid.org/0000-0002-5984-9485 \\ Doo Sang Kim https://orcid.org/0000-0001-6405-1294
}

\section{AUTHOR CONTRIBUTIONS}

Conceptualization: SHK, DSK; data curation: SHK, DSK; methodology: DSK; validation: SHK; investigation: SHK, DSK; writing-original draft: SHK, DSK; and writing-review \& editing: SHK, DSK.

\section{REFERENCES}

1. Hadidi AT. Buried penis: classification surgical approach. J Pediatr Surg 2014;49:374-9.

2. Chan IH, Wong KK. Common urological problems in children: prepuce, phimosis, and buried penis. Hong Kong Med J 2016;22:263-9.

3. Palinrungi MA, Kholis K, Syahrir S, Syarif, Faruk M. Multiple preputial stones: a case report and literature review. Int J Surg Case Rep 2020;70: 87-92.

4. Maizels M, Zaontz M, Donovan J, Bushnick PN, Firlit CF. Surgical correction of the buried penis: description of a classification system and a technique to correct the disorder. J Urol 1986;136(1 Pt 2):268-71.

5. Kon M. A rare complication following circumcision: the concealed penis. J Urol 1983;130:573-4.

6. Bhat GS. Preputial calculi: a case report and review of literature. Indian J Surg 2017;79:70-2.

7. Ellis DJ, Siegel AL, Elder JS, Duckett JW. Preputial calculus: a case report. J Urol 1986;136:464-5.

8. Zhang D, Li S, Zhang Z, Li N, Yuan X, Jia Z, et al. Urinary stone composition analysis and clinical characterization of 1520 patients in central China. Sci Rep 2021;11:6467.

9. King IC, Tahir A, Ramanathan C, Siddiqui H. Buried penis: evaluation of outcomes in children and adults, modification of a unified treatment algorithm, and review of the literature. ISRN Urol 2013;2013:109349.

10. Manasherova D, Kozyrev G, Gazimiev M. Buried penis surgical correction: midline incision rotation flaps. Urology 2020;138:174-8. 